\title{
COMMENT
}

\section{There is a sanity clause}

\author{
Gregory A Petsko*
}

Last month I tried to inject some sanity into the debate about the establishment of a new National Center for Advancing Translational Sciences (NCATS) at the National Institutes of Health (NIH). NCATS, the favored child of NIH Director Francis Collins, has as its stated scientific rationale: "to develop and offer innovative services and expertise in moving promising products through the development pipeline, as well as develop novel approaches to therapeutics development, stimulate new avenues for basic scientific discovery, and complement the strengths of existing NIH research activities". My argument, you may recall, was that it was right to worry about this new initiative to facilitate curing diseases being oversold to Congress and the US public, just as the War on Cancer and the Human Genome Project and the NIH budget doubling and the Structural Genomics Initiative and the Cancer Genomics program and genome-wide association studies have been. I pointed out that curing disease is difficult, time-consuming, and usually depends on breakthroughs that were never part of any targeted research program. I stressed the importance of funding individual investigatorinitiated, curiosity-driven projects as the essential platform on which all so-called 'translational research' research aimed at translating basic discoveries into disease cures and prevention - must be built.

But - and to the surprise of some of you from the response I got - I also said that I didn't think the formation of NCATS was necessarily a bad idea, and I promised to explain why and to offer some concrete suggestions for how it might turn into a good idea. Before I do that, I should point out that some of the flap over its creation has to do not with the idea of NCATS itself but with the fate of another NIH Center, the National Center for Research Resources (NCRR) - and yes, I know, this column really ought to be an acronym-free zone, but we're dealing with the Federal Government here, and YJBGUTI (You Just Better Get Used To It).

The total number of NIH Institutes and Centers is fixed by a 2006 federal law at 27, which is what there are

*Correspondence: petsko@brandeis.edu

Rosenstiel Basic Medical Sciences Research Center, Brandeis University, Waltham, MA 02454-9110, USA currently, so if one is to be added, something has to go. Collins decided last year to combine the National Institute on Drug Abuse and the National Institute on Alcohol Abuse and Alcoholism into a new Addictions institute, which would free up a slot, but that merger is on a long time line and, as I explained last month, Collins is in a hurry to get NCATS started. So he has proposed eliminating NCRR and, in addition to vast anxieties on the part of the people who work there, this decision has created a stir among research scientists who depend on the resources that NCRR provides, and among NIH supporters in Congress, some of whom are starting to feel that the elimination of that Center and the establishment of a new one is being done without due deliberation and consultation.

At the moment, NCRR supports all aspects of clinical and translational research, including the Clinical and Translational Science Awards, by which the Center identifies innovative research teams and equips them with essential tools and critical resources needed to tackle complex health problems. In addition, it establishes clinical research infrastructure, including specialized research staff, informatics support, and laboratories that enable studies of the full range of human disorders; it funds career development programs for medical students and physicians; and it funds development programs for underserved states and institutions that focus on health disparities that affect racial and ethnic minority populations. It also provides access to state-of-the art technologies and instruments that enable both basic biomedical research and clinical investigations of a multitude of health issues, from cancer to infectious diseases, and develops and provides access to critical animal models for a broad range of human disorders, such as Parkinson's disease, multiple sclerosis, and AIDS. It's also one of the few places that institutions can go to for funding to expand, remodel, and renovate or alter existing research facilities or construct new research facilities. In short, NCRR does quite a lot of things that are directly connected to translating discoveries into cures, but it's not clear that these programs are going to be subsumed into NCATS or that they will find homes at other Institutes and Centers where they will not become poor stepchildren. And this uncertainty is getting a number of people upset.

So the whole thing could conceivably get killed before it gets started, but I don't think that's going to 
happen. One way or another, NCATS will become a reality, because Collins has staked his prestige and legacy on its creation and there are lots of people out there who feel the NIH needs to do more to foster the development of therapies.

Besides, as I alluded to above, I don't think NCATS is a terrible idea per se. The devil, as always, is in the details, and up to now the details have been rather lacking. Which is perfect, because where there is a vacuum of information, pundits such as I can rush in with unsolicited advice. Which, again in the spirit of being sane and sober about this whole business, is what I'm now finally about to do.

If I had to summarize the non-NCRR-related objections to the new Center in a single sentence, it would be that the NIH has no business doing what pharmaceutical and biotechnology companies are supposed to do, and would probably do better. Of course, it could always be argued that there are many diseases that the private sector isn't interested in because the market is too small or the challenge is too difficult, and NCATS could simply focus on these - and that is one of the arguments that in fact is being made for its establishment. But I don't buy that rationale. Making drugs, especially small-molecule drugs, is no job for amateurs, and that's exactly what NIH is when it comes to the pharmaceutical business.

The time-line for small-molecule drug development is extremely long and the success rate is extremely low. It's also more of an art than a science. In every drug company there are literally hundreds of chemists, but only a handful of them will have ever really made a drug, and only these same handful ever will make another one - all of the rest are simply supporting the efforts of the ones who have the knack. If NCATS tries to get into the drugmaking business without a number of these especially talented chemists, the odds are they will never make one.

But this consideration does not apply to biopharmaceuticals such as protein drugs. Antibodies and hormones and other 'biologicals', as the drugs industry calls them, are preselected for efficacy by natural selection, and consequently have a much shorter time-line to the clinic and a lower failure rate in clinical trials than chemical drugs. True, after leaving this arena to the biotechnology companies for decades, the large pharmaceutical companies are now playing in it with a vengeance, but there is still plenty of room for an outsider to play in the smaller arena of rare disorders. So my first suggestion is that, if NCATS is going to try to develop drugs on its own, they need to be macromolecular drugs.

But I think an even better strategy would be for NCATS to focus, not on discovering its own pharmaceuticals, but on finding ways to overcome the roadblocks that are preventing private industry from doing that more efficiently. For example, delivery of biopharmaceuticals is often difficult, expensive, and discomforting for the patient.
Better delivery strategies would be welcome. In addition, nearly all biologicals, even those that are supposedly pure human proteins, are immunogenic to some (and sometimes many) patients. The reason is not impurities so much as the presence, in chemically pure samples, of misfolded and aggregated material, which break immune tolerance. New methods to produce biopharmaceuticals and to purify and store them while retaining conformational homogeneity would be a huge advance for the field.

Other bottlenecks are similarly easy to identify. A large percentage of drugs fail in Phase II clinical trials because they are not efficacious against the disease for which they were intended. Yet they obviously seemed to be effective in preclinical studies, and were deemed safe in people in Phase I studies. All of which suggests to me that our animal models for toxicity are pretty good, but our models for disease are not. The development of cellular and animal disease models that would allow more accurate, and earlier, target validation would enormously accelerate the translation of basic research into the clinic.

I could list several more (for example: Why do so many drugs produce peripheral neuropathy, and is there any way to either predict this in advance or to get around it? Another common side-effect of many drugs is cardiac arrythmia; why is that so, and what are the off-target macromolecules responsible for it? Can Phase II failures, which after all have been shown to be safe in people, be resurrected, Lazarus-style, for other diseases that were not their target in the first place? and so on), but you get the idea. If NCATS did nothing more but gather all the failed drugs from all the clinical trials conducted by all the pharmaceutical and biotechnology companies and make them available to researchers in universities and medical schools in the US and elsewhere, I would bet it would find several useful 'new' drugs in a matter of a few years. And as for my other ideas, well, the way to work on those is not for NCATS to do its own in-house research or to fund big drug development projects in big centers in big institutions; these are fundamental questions that need to be answered and the way to do that is with individual investigator-initiated grants. Lots of them. NCATS should pose the questions (or call them 'grand challenges' if you prefer), evaluate the proposals that come in to answer them, fund the best, and then get out of the way.

In other words, the best strategy for NCATS to make a real difference in translational research is, ironically, for it to fund a lot of basic research. I know that sounds crazy, but then, there's a fine line between genius and insanity, and maybe it's time we erased it.

Published: 25 February 2011

doi:10.1186/gb-2011-12-2-105

Cite this article as: Petsko GA: There is a sanity clause. Genome Biology 2011 12:105. 